was lost after the conclusion of the War in resuming investigations.

Vol. I of Series 2 contains botanical papers published between I92I and I924. To the botanists in a new country falls the duty of widening our horizon as to botanical conditions, and these papers are valuable examples of such literature. They include ecological studies of Australian vegetation by Marjorie I. Collins, of mangrove and salt marsh vegetation and arid and semi-arid regions; studies of Australian parasitic flowering plants, and of Australian examples of symbiosis (mycorhiza and root-nodules) by J. M'Luckie, also notes upon the life-history and structure of Australian Phanerogams and ferns by Prof. Lawson, P. Brough, and Jessie Steele. M. M. Williams has a brief note upon interesting brown seaweeds, including the one apparent parasite in the group, Notheia anomala.

Series II covers mathematics, physics, and astronomy, and vol. I contains seven papers dealing mainly with physical questions. They show that the University is not restricting its research work to the industrial problems which arise in the colony, but is taking its part in the solution of scientific questions the interest of which is world wide. Mr. E.H. Booth deals with the ions produced in air which has bubbled through water, a process which bears on the problem of how the earth maintains its negative charge. Dr. G. Harker shows that the vapour arising from a boiling solution has a temperature higher than that from the pure solvent boiling under the same conditions. Prof. Carslaw solves a problem on the cooling of a sphere with a core and shows that in the Mathematical Tripos of I9O4 the candidates were asked to prove something which was untrue, while a paper by the late Prof Pollock gives an interesting account of the physics of the stethoscope.

Vol. I of Series 3 is a collection of papers published in the various chemical journals by workers in the chemistry departments of the University. These show that research work is being actively prosecuted. Physical chemistry measurements include those of the molecular solution volumes of various organic substances in ethyl alcohol, which show that several of the solutes are associated in the liquid state; the development of the miscibility tests for eucalyptus oils in which the oils are added to methyl alcoholwater mixtures and the critical solution temperatures determined; the decrease in the values of the reaction constant in the decomposition of hydrogen peroxide by colloidal platinum when different concentrations of protective colloids are added, and the action of soaps as protective colloids for gold hydrosols, in which it is shown that although the stabilisation is very pronounced the coagulating influence of the sodium ion of the lower soaps begins to outweigh the protective action. A number of the reprints deal with organic chemistry: the preparation of organic compounds of arsenic of the type $R_{1} R_{2} R_{3} A s$, $R A s I_{2}$ where $R_{1} R_{2}$, etc., indicate similar or dissimilar alkyl or aryl groups; studies in the formation of ring compounds by condensation of aromatic amines and diketones, etc. ; researches on the phellandrenes and a number of contributions to the chemistry of piperitone. In addition, investigations have been carried out on the active principle of trees of the kind to which belong the dreaded "ordeal " or "doom tree" of the African pygmies.

\title{
Voltaire and Medicine.
}

I the second part ${ }^{1}$ of his paper on "Voltaire and Medicine," read before the Section of the History of Medicine of the Royal Society of Medicine on December 16 , the president, Dr. J. D. Rolleston, gave some account of Voltaire's allusions to anatomy and physiology, his advocacy of inoculation against small-pox, and his interest in the history and ravages of syphilis, a knowledge of which he had derived from Astruc's work on venereal diseases. $\mathrm{He}$ also referred to the attention paid by Voltaire to other matters connected with public health, his acquaintance with medical jurisprudence and particularly his sceptical attitude toward historical cases of poisoning, in many of which he showed that death was more probably due to some acute infection, and his remarks on various diseases of social importance, such as mental disorders, convulsive hysteria at the tombs of saints and ecclesiastics, alcoholism and the King's evil.

Several passages in his works show that Voltaire had paid some attention to anatomy. He commences his article on "Man" in the "Dictionnaire Philosophique " by saying that in order to learn the physical aspects of the human race, one must study works of anatomy or the article of M. Venel in the "Dictionnaire Encyclopédique," or rather follow a course of anatomy. In his article on "Anatomy" in the same work, he remarks that "Ancient anatomy bears to modern anatomy the same relation that the rough geographical charts of the sixteenth century do to the topographical maps of to-day." In the "Histoire du Docteur Akakia" Maupertuis is condemned for his advice to neglect anatomy. Voltaire, however, fully realised that much was yet learned

1 See Nature, December I9, I925, p. 9 Ig. in human anatomy. In Chap. 35, entitled "Incertitudes en Anatomie" of the essay "Des singularités de la nature," he exclaims, "In spite of all the help that the microscope has given anatomy, and in spite of the great discoveries of so many skilful surgeons and physicians, what interminable discussions have arisen, and in what uncertainty we still remain !',

Although physiology was still in its infancy in the eighteenth century, Voltaire seems to have been well abreast of the knowledge of his time. In the " Dictionnaire Philosophique" he alludes to the uncertainties current in connexion with circulation, digestion, generation, and muscular tonicity, and refers to the pioneer work of Borelli, Keil and Jurin in cardiac physiology. He exposes the errors of Descartes regarding digestion and circulation, and heaps ridicule on the casuist Sanchez and the philosopher Maupertuis for their extraordinary views on generation and embryology.

Voltaire's interest in public health was shown not only by his desire to control small-pox by inoculation, and syphilis by a league of nations to combat the disease-a project not realised until the formation of the Union internationale contre le péril vénérien in I923-but also by his allusion to other epidemic diseases, especially plague and malaria, and his condemnation of the insanitary condition of the Paris hospitals, in which overcrowding and cross infection were rife. He also showed his concern for public health in his references to the abuses connected with the administration of military hospitals, the congested state of the Paris cemeteries and the practice of burial in churches, as well as by his proposal to found maternity hospitals for unmarried women. 